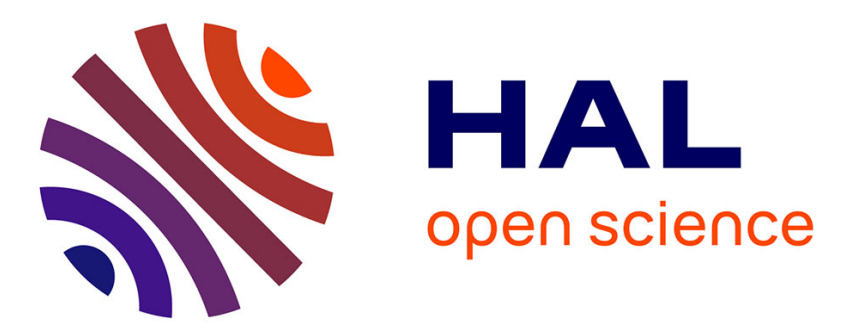

\title{
Reciprocity-Based Cognitive Transmissions using a MU Massive MIMO Approach
}

\author{
Boris Kouassi, Irfan Ghauri, Luc Deneire
}

\section{To cite this version:}

Boris Kouassi, Irfan Ghauri, Luc Deneire. Reciprocity-Based Cognitive Transmissions using a MU Massive MIMO Approach. IEEE International Conference on Communications (ICC), 2013 (Cognitive Radio and Networks Symposium), Jun 2013, Budapest, Hungary. pp.1331-1335. hal-00845407

\section{HAL Id: hal-00845407 \\ https://hal.science/hal-00845407}

Submitted on 17 Jul 2013

HAL is a multi-disciplinary open access archive for the deposit and dissemination of scientific research documents, whether they are published or not. The documents may come from teaching and research institutions in France or abroad, or from public or private research centers.
L'archive ouverte pluridisciplinaire HAL, est destinée au dépôt et à la diffusion de documents scientifiques de niveau recherche, publiés ou non, émanant des établissements d'enseignement et de recherche français ou étrangers, des laboratoires publics ou privés. 


\title{
Reciprocity-Based Cognitive Transmissions using a MU Massive MIMO Approach
}

\author{
Boris Kouassi ${ }^{\dagger}$, Irfan Ghauri ${ }^{\ddagger}$, Luc Deneire ${ }^{\dagger}$ \\ ${ }^{\dagger}$ Laboratoire I3S, Sophia-Antipolis, France. Email: \{kouassi, deneire $\} @ i 3 s . u n i c e . f r$ \\ ${ }^{\ddagger}$ Intel Mobile Communications, Sophia-Antipolis, France. Email: irfan.ghauri@intel.com
}

\begin{abstract}
Cognitive radio (CR) aims at enabling the coexistence between legacy systems called primary users (PU) and secondary users (SU), provided the $\mathrm{SU}$ do not (significantly) cause interference on PU links. Massive MIMO systems on the other hand yield significant performance and reliability enhancement. In this paper, we investigate the capacity of both $\mathrm{PU}$ and $\mathrm{SU}$ systems using multi-user (MU) massive MIMO and time division duplex (TDD). TDD indeed permits to estimate the channel state information (CSI) at the PU/SU transmitters to perform linear precoding, taking advantage of the channel reciprocity. The capacity is derived considering the CSI imperfections, the nonreciprocal radio frequency front-ends as well as antenna coupling effects. These impairments can be compensated using reciprocity calibration techniques we recently developed. Extending these techniques to MU massive MIMO, we evaluate interference cancellation precoders. Simulations reveal performance improvements using the massive MIMO reciprocity-based precoder in a CR approach.
\end{abstract}

Index Terms-Massive MIMO, cognitive radio, reciprocitycalibration, beamforming, precoding, channel estimation.

\section{INTRODUCTION}

The spectrum scarcity generated by the evolution of wireless technologies this last decade leads to rethink transmissions strategies. Therefore, for a smarter utilization of the spectrum, cognitive radio $(\mathrm{CR})$ has been developed. It exploits radio parameters like spectral occupancy, waveform, etc, to enable unlicensed transmissions (secondary: SU), while avoiding disrupting licensed systems (primary: PU) [1].

In this study the PU/SU coexistence is achieved based on a massive MIMO linear precoding. The massive MIMO has been introduced by Marzetta in [2], and suggests to use a large antenna array at the base stations (BS), to reduce uncorrelated noise and fast fading effects [3], [4], [5]. Recent investigations based on smart partitioning of users or random matrix theory have shown that the number of antennas needed to benefit from the "massive MIMO effect" is reasonable (about 50, see e.g. [6], [4]).

In this paper, we aim at enhancing SUs transmissions without harming legacy communications in a CR context, therefore, we design a linear precoder (beamforming [7]) exploiting the interfering channels between the SU transmitter (Tx) and the PU receiver $(\mathrm{Rx})$. This idea has been proposed in [8], where authors suppose a training phase to learn the interfering channels before transmitting in the PUs white spaces. The estimation of crosslink channels is a challenge in $\mathrm{CR}$, since generally there is no cooperation between PUs and SUs. Accordingly, we assume that the SU estimates the reverse crosslink channel using the training sequences broadcast by PUs, then the forward channel is found helped by the channel reciprocity assumption. This reciprocity hypothesis is abusively assumed in most massive MIMO TDD (time division duplex) key studies (see e.g. [6], [2], [5]). Indeed the overall channel including $\mathrm{Tx} / \mathrm{Rx}$ filters is not reciprocal due to the radio frequency front-ends (RF) circuitry and because large amount of antennas leads to close antennas spacing. This inevitably leads to mutual coupling such that current induced on one antenna impacts nearby antennas, thus causing breakdown to the fundamental reciprocity assumption required to design the precoder [9], [10], [11]. Reciprocitycalibration methods have been recently proposed (e.g. [10], [11]) and implemented [12] for conventional MIMO systems and are not always relevant to multiple user massive MIMO. A recent work [13] addresses the calibration in massive MIMO, but authors did not consider coupling effects. The main contribution of this paper is to address properly the impact of RF impairments, then define a massive MIMO reciprocity-calibration considering the antenna coupling, and finally investigate the performances of the massive MIMO precoding approach in CR transmissions.

Our work is structured as follows: Section II describes the CR scenario and backgrounds. In Section III we illustrate the RF impacts and Section IV proposes a massive MIMO calibration approach. Section V shows the transmission strategy, evaluation framework and the simulation results. Finally, section VI draws conclusions.

\section{TRANSMISSION SYSTEM AND BACKGROUND}

\begin{tabular}{ll} 
& Table I: notations \\
\hline Symbol & Description \\
\hline$*$ & Convolution \\
$\otimes$ & Kronecker product \\
$c, \mathbf{v}, \mathbf{M}$ & Complex or real scalar, vector, matrix \\
$\mathbf{M}^{T}$ & Transpose matrix of $\mathbf{M}$ \\
$\mathbf{M}^{*}$ & Conjugate matrix of $\mathbf{M}$ \\
$\mathbf{M}^{\dagger}$ & Hermitian matrix of $\mathbf{M}$ \\
$v e c(\mathbf{M})$ & Vectorization of matrix $\mathbf{M}$ \\
$\mathbf{F} \mathbf{T}^{-1}\{\mathbf{M}(\nu)\}=\mathbf{M}(\tau)$ & Inverse discrete Fourier transform of $\mathbf{M}(\nu)$ \\
\hline
\end{tabular}

Throughout this paper, we use the notations in Table I. We consider a multipath $M \times N$-MIMO (multiple input multiple output) channel with $P$ paths each having its own delay $(\tau)$ and attenuation. The time domain received signal $\mathbf{y}(t) \in \mathbb{C}^{N \times 1}$ from a base station to user is the convolution of the lines in the multipath channel matrix $\mathbf{C}(t, \tau) \in \mathbb{C}^{N \times M}$ with the vector 
transmitted by $M$ antennas $\mathbf{x}(t) \in \mathbb{C}^{M \times 1}$ such:

$$
\mathbf{y}(t)=\left[\begin{array}{lll}
y_{1}(t) & \cdots & y_{N}(t)
\end{array}\right]^{T}=\mathbf{C}(t, \tau) * \mathbf{x}(t)+\mathbf{n}(t),
$$

with $\mathbf{n}(t) \in \mathbb{C}^{N \times 1}$ the additive white Gaussian noise (AWGN) at the receiver $(\mathrm{Rx})$. The cognitive radio scenario is defined in Fig. 1 by a primary and a secondary cell with respectively $K$ and $L$ users, $N_{p}, N_{s} \leq 2$ antennas per user and $M_{p}, M_{s}$ antennas respectively at the primary $\left(P_{B S}\right)$ and the secondary base stations $\left(S_{B S}\right)$. They use the same bandwidth in TDD mode with an orthogonal frequency division multiplexing (OFDM) and all users share the same time/frequency resource (MU-MIMO). In order to transmit in the SU without disrupting

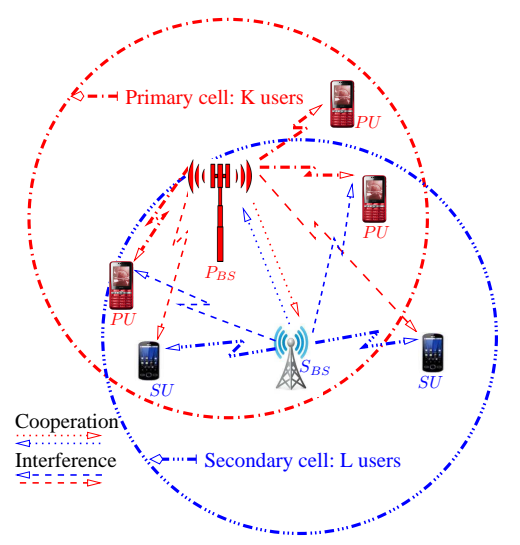

Fig. 1. MU MIMO CR scheme with PU and SU users.

the PU, we exploit the spatial degrees of freedom $(d)$ in MIMO systems related to the network capacity $C$ such: $C(S N R)=$ $d \log (S N R)+o(\log (S N R))$ [14]. Like described in [15], in our CR scenario with interfering channels in Fig.1, $d=$ $\min \left(K N_{p}+L N_{s}, M_{s}+M_{p}, \max \left(M_{p}, L N_{s}\right), \max \left(M_{s}, K N_{p}\right)\right)$, thus increasing the number of antennas also increases $d$ and consequently the channel capacity.

In this paper, the transmission strategy is drawn from the massive MIMO concept using a large number of BS antennas $M_{p}, M_{s} \mapsto \infty$ [5], [4]. In an independent and identically distributed (i.i.d) complex Gaussian channel, the achievable downlink (DL) ergodic capacity in the PU cell $\left(C_{p}\right)$ with perfect channel state information at the receiver (CSIR) is expressed in the frequency domain by:

$$
\begin{gathered}
C_{p}=\mathbb{E}\left[\log _{2}\left\{\operatorname{det}\left(\mathbf{I}_{N_{p}}+\mathbf{R}_{s p}^{-1} \mathbf{G}_{p p} \boldsymbol{\Phi}_{p} \mathbf{G}_{p p}^{\dagger}\right)\right\}\right], \\
\text { s.t. } \operatorname{tr}\left(\boldsymbol{\Phi}_{p}\right) \leq \phi_{p},
\end{gathered}
$$

with $\mathbb{E}[\bullet]$ the expectation, $(\bullet)^{-1}$ the (pseudo)inverse, $\boldsymbol{\Phi}_{p}=$ $\mathbb{E}\left[\mathbf{x}_{p} \mathbf{x}_{p}^{\dagger}\right]$ denotes for the power allocation matrix at $P_{B S}$, $\left(\phi_{p}, \phi_{s}\right)$ the power constraints at $P_{B S}$ and $S_{B S}, \mathbf{x}_{p} \in \mathbb{C}^{M_{p}}$, $\mathbf{x}_{s} \in \mathbb{C}^{M_{s}}$ the transmitted vectors, $\mathbf{R}_{s p}=\mathbf{G}_{s p} \mathbf{x}_{s}\left(\mathbf{G}_{s p} \mathbf{x}_{s}\right)^{\dagger}+$ $\sigma_{n} \mathbf{I}_{N_{p}}$ the interference plus noise matrix from $S_{B S}$, with $\mathbf{G}_{p p}=\left[\mathbf{G}_{p 1}, \ldots, \mathbf{G}_{p K}\right], \mathbf{G}_{p 1} \in \mathbb{C}^{N_{p} \times M_{p}}$ the PU DL channel, and $\mathbf{G}_{s p}$ the DL channel from SU to PU. The CR problem can be reformulated as a constrained maximization:

$$
\begin{gathered}
\max _{\mathbf{P}_{s}, \mathbf{P}_{p}}\left(C_{s}=\log _{2}\left\{\operatorname{det}\left(\mathbf{I}_{N_{s}}+\mathbf{R}_{p s}^{-1} \mathbf{G}_{s s}\left(\mathbf{P}_{s} \mathbf{\Phi}_{s} \mathbf{P}_{s}^{\dagger}\right) \mathbf{G}_{s s}^{\dagger}\right\}\right),\right. \\
\text { s.t. } \mathbf{G}_{s p} \mathbf{x}_{s}=\mathbf{0}, \operatorname{tr}\left(\mathbf{P}_{s} \boldsymbol{\Phi}_{s} \mathbf{P}_{s}^{\dagger}\right) \leq \phi_{s},
\end{gathered}
$$

with $\mathbf{R}_{p s}$ the interference plus noise covariance matrix from PU to SU. This corresponds to the spatial interweave CR approach which transmits in spatial holes using a precoder $\mathbf{P}_{s}$. Consequently, in order to steer null interferences toward the PU while improving SU transmissions, we perform the CR scenario based on the PU and SU beamformers (precoders) $\mathbf{P}_{p}$ and $\mathbf{P}_{s}$. Assuming first that the channels are perfectly estimated, $\mathbf{P}_{p}$ is designed using the PU DL channel estimate $\hat{\mathbf{G}}_{p p}$, and $\mathbf{P}_{s}$ from the knowledge of the interfering DL estimation $\hat{\mathbf{G}}_{s p}$. The $S_{B S}$ signal is projected on an orthogonal basis of $\mathbf{G}_{s p}$ using the singular value decomposition of $\hat{\mathbf{G}}_{s p}$ $\mathbf{U D V}^{\dagger}=\operatorname{SVD}\left\{\hat{\mathbf{G}}_{s p}\right\}$, such that the signal from $S_{B S}$ to PU is automatically canceled. The kernel $\mathbf{W}=\operatorname{Ker}\left\{\hat{\mathbf{G}}_{s p}\right\}$ is spanned by the last columns of $\mathbf{V}$, leading to the following interference cancellation precoders:

$$
\mathbf{P}_{p}=\sqrt{\frac{\phi_{p}}{\operatorname{tr}\left(\hat{\mathbf{G}}_{p p}^{\dagger} \hat{\mathbf{G}}_{p p}\right)}} \hat{\mathbf{G}}_{p p}^{\dagger}, \mathbf{P}_{s}=\sqrt{\frac{\phi_{s}}{\operatorname{tr}\left(\mathbf{G}_{\mathbf{w}}^{\dagger} \mathbf{G}_{\mathbf{w}}\right)}} \mathbf{G}_{\mathbf{w}}
$$

with $\mathbf{G}_{\mathbf{w}}=\left(\mathbf{W} \mathbf{W}^{\dagger} \hat{\mathbf{G}}_{s s}^{\dagger}\right)$. Moreover, in multi-cell system with $B$ BS and $K$ users, the frequency domain DL signal in a cell $b$ is given by:

$$
\mathbf{Y}_{K}=\mathbf{G}_{b} \mathbf{X}_{b}+\sum_{c=1, c \neq b}^{B} \mathbf{G}_{c} \mathbf{X}_{c}+\mathbf{N}_{K}
$$

with $\mathbf{Y}_{K}=\left[\mathbf{y}_{1}, \ldots, \mathbf{y}_{K}\right], \mathbf{G}_{b}=\left[\mathbf{G}_{b 1}, \ldots, \mathbf{G}_{b K}\right]$ the DL channel in cell $b, \mathbf{N}_{K}$ the noise matrix at $\mathrm{Rx}$ and $\sum_{c=1, c \neq b}^{B} \mathbf{G}_{c} \mathbf{X}_{c}$ the interfering signal from other BSs. Using the relation (5), the received signals $\mathbf{y}_{p}$ and $\mathbf{y}_{s}$ at the PU and $\mathrm{SU}$ in our CR scenario yield:

$$
\begin{aligned}
& \mathbf{y}_{p}=\mathbf{G}_{p p} \hat{\mathbf{G}}_{p p}^{\dagger} \mathbf{x}_{p}+\mathbf{G}_{s p}\left(\mathbf{W} \mathbf{W}^{\dagger} \hat{\mathbf{G}}_{s s}^{\dagger}\right) \mathbf{x}_{s}+\mathbf{n}_{p}, \\
& \mathbf{y}_{s}=\mathbf{G}_{s s}\left(\mathbf{W} \mathbf{W}^{\dagger} \hat{\mathbf{G}}_{s s}^{\dagger}\right) \mathbf{x}_{s}+\mathbf{G}_{p s} \hat{\mathbf{G}}_{p p}^{\dagger} \mathbf{x}_{p}+\mathbf{n}_{s} .
\end{aligned}
$$

We observe that the CSIs at $\mathrm{Tx} / \mathrm{Rx}$ are required for the precoding and coherent detection, therefore pilot training symbols $\left(\mathbf{p}_{u l / d l}\right)$ known at both side $(\mathrm{Tx} / \mathrm{RX})$ are periodically emitted in one OFDM symbol, then the estimated channel persubcarrier is exploited for detection in the following symbols according to the channel coherence time. The uplink (UL) channel $(\hat{\mathbf{H}})$ is estimated at BSs using a minimum mean squared error estimator (MMSE extensively studied e.g. [16]), while the DL estimation process can be simplified at the user side using a least squares approach.

Note that PU and SU use the same pilot structure, in order to avoid pilot contamination, the $P_{B S}$ broadcasts periodically control signals specifying the pilot parameters to $S_{B S}$ (see Fig. 1). Nevertheless, PU users are not able to estimate and feedback $\mathbf{G}_{s p}$ to $S_{B S}$ for Tx-beamforming, thereby we use the UL interfering channel $\mathbf{H}_{p s}$ acquired from PU training sequences, and find the DL $\mathbf{G}_{s p}$ using the channel reciprocity assumption in TDD. However, the overall channel including $\mathrm{Tx} / \mathrm{Rx}$ filters in Fig. 2 are not reciprocal due to the RF imperfections and mutual coupling [9], [10]. The next section addresses the RF impacts and solution for massive MIMO. 


\section{RADIO FREQUENCY (RF) AND COUPLING EFFECTS}

Fig. 2 illustrates the RF basic components encountered in TDD MU-MIMO which generate perturbations like antenna gain imbalance, frequency error, noise from analog to digital converters, etc. Though some of these non-exhaustive impairments are commonly addressed in nowadays systems, they destroy the channel reciprocity assumption. Motivated by [11],

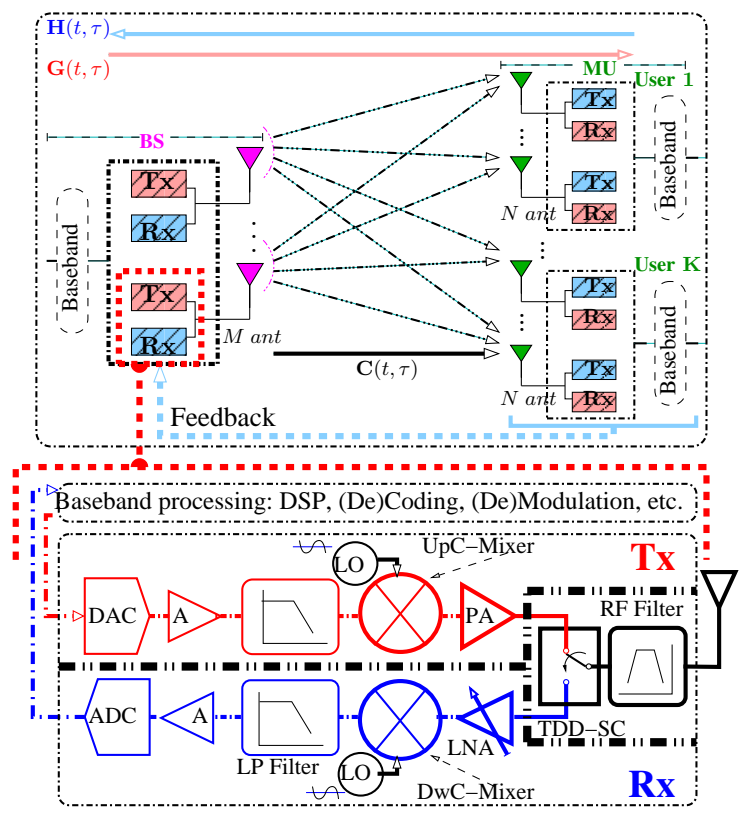

Fig. 2. Tx/Rx RF filters in the MU-TDD system composed by: the switching circuit (TDD-SC), a low noise amplifier (LNA), a down/up-converter (DwCMixer), a local oscillator (LO), a low pass (LP) filter, power amplifiers (PA, A), a digital (analog) to analog (digital) converter (DAC, ADC).

where the RF front-ends are assumed frequency selective, we model RF impairments by matrices. Then the non-reciprocal UL/DL channels including the RF in Fig.2 are described by:

$$
\begin{aligned}
& \mathbf{H}(t, \tau)=\mathbf{R}_{B S}(t, \tau) * \mathbf{C}^{T}(t, \tau) * \mathbf{T}_{M U}(t, \tau), \\
& \mathbf{G}(t, \tau)=\mathbf{R}_{M U}(t, \tau) * \mathbf{C}(t, \tau) * \mathbf{T}_{B S}(t, \tau),
\end{aligned}
$$

where the square $M \times M$ matrices $\mathbf{R}_{B S}(t, \tau), \mathbf{T}_{B S}(t, \tau)$ stand for the Tx/Rx filters at the BS, $\mathbf{R}_{M U}(t, \tau), \mathbf{T}_{M U}(t, \tau)$ the $K N \times$ $K N$ matrices containing the users RF filters and $\mathbf{C}(t, \tau)$ the reciprocal propagation channel between antennas.

Throughout this paper, we suppose the RF time-invariant (i.e. they vary more slowly than the channel) because the electronic components properties vary in specific conditions like the temperature [9]. We assume the users randomly distributed in the cells and separated by several wavelengths $(\lambda \approx 10 \mathrm{~cm}$ for $\mathrm{GHz}$ signals). In the current state of technology, most of the MIMO techniques (beamforming, space-time coding, etc) require an antenna separation close to $\lambda / 2$. The difficulty to get a suitable separation in perfect isolation induces coupling effects in massive MIMO. The coupling matrix from close $M$ antennas is modeled in [17] by $\mathbf{C}_{\mathbf{P}}=\left(z_{I}+z_{T}\right)\left(\mathbf{Z}+z_{T} \mathbf{I}_{M}\right)^{-1}$, where $z_{I}$ and $z_{T}$ are the antennas impedance in isolation and at the receiver, $\mathbf{Z}$ the mutual impedance matrix whose elements depend on the antenna's geometry. Ideally, the RF matrices are all supposed diagonal. But as shown in [17], the coupling effects lead to full RF matrices at BS side and diagonal block matrices at the user side, since the distance between users is supposed large enough. Then, from (7) the overall DL is:

$$
\mathbf{G}(t, \tau)=\mathbf{Q}_{M U}(\tau) * \mathbf{H}^{T}(t, \tau) * \mathbf{Q}_{B S}(\tau) .
$$

Writing $\mathbf{Q}_{M U}(\tau)=\mathbf{F T}^{-1}\left\{\mathbf{Q}_{M U}(\nu)\right\}$ and $\mathbf{Q}_{M U}(\nu)=$ $\mathbf{R}_{M U}(\nu) \mathbf{T}_{M U S}^{-T}(\nu), \mathbf{Q}_{B S}(\nu)=\mathbf{R}_{B S}^{-T}(\nu) \mathbf{T}_{B S}(\nu), \nu$ the subcarrier subscript, the relation (8) in frequency domain is:

$$
\mathbf{Q}_{M U}^{-1}(\nu) \mathbf{G}(t, \nu)=\mathbf{H}^{T}(t, \nu) \mathbf{Q}_{B S}(\nu) .
$$

The next section describes a solution to compensate the RF impairments and to restore the channel reciprocity.

\section{RECIPROCITY-CALIBRATION}

The literature illustrates some conventional MIMO calibration approaches [11], [9], but implementing such method in massive MIMO is constraining due to the large amount of MU CSI. However, the flexibility of relative calibration has been adopted in this work, it does not require any third-party or hardware modifications (unlike absolute calibration) and simply exchanges the CSI at the two ends of the link. We propose to calibrate independently each user with the BS, namely a per-user calibration. Thereby, each user calibration is a MIMO calibration mentioned in [11]. Consequently, using the frequency domain formulation (9), the calibration consists in finding the RF matrices minimizing the following distance:

$$
\min _{\left\{\mathbf{Q}_{M U}, \mathbf{Q}_{B S}\right\}}\left\|\operatorname{vec}\left(\mathbf{Q}_{M U}^{-1}(\nu) \mathbf{G}(t, \nu)\right)-\operatorname{vec}\left(\mathbf{H}^{T}(t, \nu) \mathbf{Q}_{B S}(\nu)\right)\right\|^{2} .
$$

We have shown in [11], that the relation (10) can be rewritten as a total least squares (TLS) problem:

$$
\begin{aligned}
& \min _{\left\{\mathbf{q}_{M B}, \boldsymbol{\Delta} \mathbf{A}_{\mathbf{T}}\right\}}\left\|\boldsymbol{\Delta} \mathbf{A}_{\mathbf{T}}\right\|_{F} \\
& \text { s.t }\left(\hat{\mathbf{A}}_{\mathbf{T}}+\boldsymbol{\Delta} \mathbf{A}_{\mathbf{T}}\right) \mathbf{q}_{M B}(\nu)=\mathbf{0}_{(T . M . N) \times 1} \text {, }
\end{aligned}
$$

$\mathbf{q}_{M B}(\nu)=\left[\begin{array}{c}\operatorname{vec}\left(\mathbf{Q}_{M U}^{-1}(\nu)\right) \\ \operatorname{vec}\left(\mathbf{Q}_{B S}(\nu)\right)\end{array}\right], \hat{\mathbf{A}}_{\mathbf{T}}=\left[\begin{array}{lll}\hat{\mathbf{A}}(1, \nu) & \cdots & \hat{\mathbf{A}}(T, \nu)\end{array}\right]^{T}$ with $\left.\hat{\mathbf{A}}=\left[\left(\hat{\mathbf{G}}^{T}(t, \nu) \otimes \mathbf{I}_{N}\right)\right)-\left(\mathbf{I}_{M} \otimes \hat{\mathbf{H}}^{T}(t, \nu)\right)\right], \hat{\mathbf{H}}, \hat{\mathbf{G}}$ the estimated UL/DL channels per user, $\Delta \mathbf{A}_{\mathbf{T}}$ the compensation matrix of the UL/DL channels estimation error. Using $\operatorname{SVD}\left\{\mathbf{A}_{\mathbf{T}}\right\}=\mathbf{U D V}^{\dagger}$, the TLS solution $\left(\hat{\mathbf{q}}_{M B}(\nu)\right)$ lies in the last column of $\mathbf{V}$ like illustrated in [18], [11].

We observe that the TLS system (11) is over-parametrized using $T$ versions of UL/DL channels over the time (where $T$ can be kept small in time domain calibration case see e.g [11]). Therefore, a short per-subcarrier feedback is assumed in order to retransmit accurately the overall $T \operatorname{DL} \operatorname{CSI}\left(\mathbf{G}(t, \nu)_{K N \times M}\right)$ from users to BS. Thereby, calibration accuracy depends as well on this feedback efficiency. In [19], authors proposed a massive MIMO feedback approach using a code-division multiple-access (CDMA) technique to project the $N K \times M$ matrices into unitary $M \times N_{u}$ spread matrices mutually orthogonal with $N_{u} \geq K . M$. In this paper, we assume no feedback delay and interferences, then the DL-CSI is directly re-modulated by users. The preliminary coding scheme is achieved using the waveform properties of Zadoff-Chu sequences $\mathbf{z}_{\mathbf{c}}(r, \alpha)=$ $e^{\left(-j \pi r \alpha \frac{(\alpha+1)}{N_{z}}\right)}$ with constant amplitude zero auto-correlation 
(CAZAC), where $\alpha, r \in\left[0, \ldots, N_{z}-1\right]$ and $N_{z}$ the length of the sequence. Accordingly, the CSI is encoded using a $M \times K N_{z}$ matrix (instead of $M \times N_{u}, N_{u} \geq K . M$ ) and we can use shorter sequences meeting $N_{z}<<M$. A root number $r$ can be assigned to each user $\left(r \leq K<N_{z}-1\right)$ such that we retrieve a specific user signal among several based on the CAZAC properties. However sequences have to be carefully selected to keep the orthogonality like illustrated in Fig. 3, where we plot the inter/auto-correlation of users 3,4 and 12 ( $r=\{3,4,12\}, N_{z}=32, M=100, N=1$ and $K=14$ users). Fig. 3 shows that users do not interfere (zero inter-correlation).
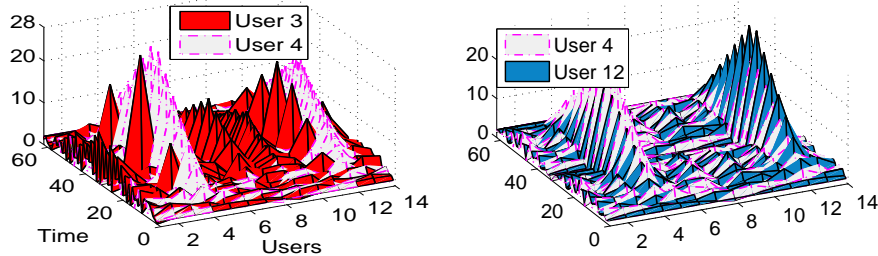

Fig. 3. Auto/inter-correlations of user 3, 4 and 12 versus the time

Although we observe a peak in inter-correlation of user 4 at the user location 12 , which may suggest interferences, however the peaks of users 4 and 12 are located at a different time, which attests no mutual interferences. Subsequently, investigations with time domain calibration mentioned in [11] are on going to address efficiently the coupling effects at the user side.

\section{Cognitive Radio Transmission Strategy}

This section describes the CR transmission strategies based on calibration, cooperation and beamforming (see Fig. 4). Assuming per-subcarrier operations, the time/frequency subscript $(t, \nu)$ will be ignored. We consider that each system PU/SU is calibrated individually (see Fig. 4) and the BS know the RF matrices of their respective users. Drawn from the equation (4), we design the beamformers $\mathbf{P}_{p}$ in $P_{B S}$ and $\mathbf{P}_{s}$ in $S_{B S}$ using the DL channels $\hat{\mathbf{G}}_{p p}, \hat{\mathbf{G}}_{s p}$ estimated after the reciprocity-calibration. Note that, $P_{B S}$ transmits

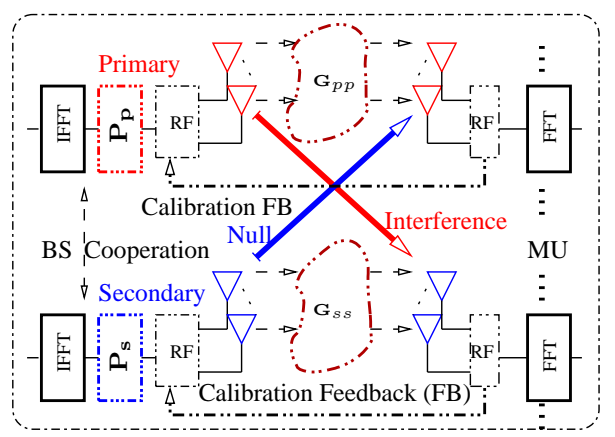

Fig. 4. Illustration of the OFDM transmission system with $\mathbf{P}_{p}, \mathbf{P}_{s}, \mathrm{BS}$ cooperation, the calibration, and the (inverse) fast Fourier transform: (I)FFT.

regardless of the secondary, but broadcasts the pilots references to prevent pilot contamination. As in [8], we suppose that the SU is aware of PU specifications, then $S_{B S}$ listens the PU UL transmissions and estimates the UL crosslink channel $\hat{\mathbf{H}}_{p s}=\mathbf{Q}_{S_{B S}}^{-T} \mathbf{G}_{s p}^{T} \mathbf{Q}_{M U p}^{-T}+\mathbf{n}_{u l}$ ( $\mathbf{n}_{u l}$ the estimation error). Nonetheless, the crosslink DL channel $\mathbf{G}_{s p}$ is not entirely estimated by the $S_{B S}$, since based on equation (8), $\mathbf{G}_{s p}=\mathbf{Q}_{M U p} \mathbf{H}_{p s}^{T} \mathbf{Q}_{S_{B S}}$, where only $\mathbf{Q}_{S_{B S}}$ is estimated by $S_{B S}$ in the SU calibration step. $S_{B S}$ has no knowledge about the PU RF matrix $\mathbf{Q}_{M U p}$. We introduced a signaling in the PU frame structure (see Fig. 5), such that PU users periodically send special pilots $\left(\mathbf{p}_{S u l}=\hat{\mathbf{Q}}_{M U p}^{T} \mathbf{p}_{u l}\right)$ with a compensation of RF impairments determined after PU calibration. Thus, using $\mathbf{y}_{s}=\mathbf{H}_{p s} \mathbf{p}_{S u l}+\mathbf{n}=\left(\mathbf{Q}_{S_{B S}}^{-T} \mathbf{G}_{s p}^{T} \mathbf{Q}_{M U p}^{-T}\right)\left(\hat{\mathbf{Q}}_{M U p}^{T} \mathbf{p}_{u l}\right)+\mathbf{n}$, the $S_{B S}$ determines the overall crosslink channel $\mathbf{G}_{s p}$ as:

$$
\begin{aligned}
\hat{\mathbf{G}}_{s p}^{T} & =\hat{\mathbf{Q}}_{S_{B S}}^{T}\left(\mathbf{Q}_{S_{B S}}^{-T} \mathbf{G}_{s p}^{T}\left(\Delta \mathbf{Q}_{M U p}\right) \mathbf{p}_{u l}\right) \mathbf{p}_{u l}^{\dagger}+\mathbf{n}_{Q p}, \\
& =\left(\Delta \mathbf{Q}_{S_{B S}} \mathbf{G}_{s p}^{T} \Delta \mathbf{Q}_{M U p}\right)\left(\mathbf{p}_{u l} \mathbf{p}_{u l}^{\dagger}\right)+\mathbf{n}_{Q p} .
\end{aligned}
$$

The interesting point is that $\mathbf{G}_{s p}$ is estimated at $S_{B S}$ only

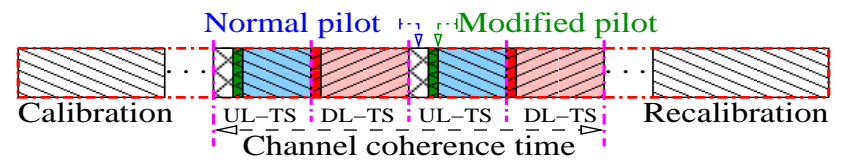

Fig. 5. The TDD frame structure in PU with the modified pilot location in the UL time slots (TS), This modified pilot is broadcast periodically such a manner that the $S_{B S}$ estimates the crosslink channel.

with the SU RF matrices. Nevertheless, a calibration error from $\mathbf{Q}_{S_{B S}}$ and $\mathbf{Q}_{M U p}$ can affect $\hat{\mathbf{G}}_{s p}$ at $S_{B S}$ and finally deteriorate the PU/SU capacity. These considerations and the transmission strategy will be evaluated in the next section.

\section{A. Numerical Results}

In this section, we evaluate the performances of the CR scenario. UL/DL channels are all supposed i.i.d complex Gaussian with an attenuation factor in the frequency domain. We assume the same channel estimation error in $\mathrm{PU}$ and $\mathrm{SU} \mathbf{n}_{e} \sim \mathcal{C N}\left\{\mathbf{0}, \sigma_{e}^{2} \mathbf{I}\right\}$. Using equation (2), the achievable ergodic DL channel capacity at $\mathrm{PU}\left(C_{p}\right)$ assuming imperfect CSIR is given by: $\quad C_{p}=\mathbb{E}\left[\log _{2}\left\{\operatorname{det}\left(\mathbf{I}_{N_{p}}+\mathbf{R}_{s p}^{-1} \hat{\mathbf{G}}_{p p}\left(\mathbf{P}_{p} \boldsymbol{\Phi}_{p} \mathbf{P}_{p}^{\dagger}\right) \hat{\mathbf{G}}_{p p}^{\dagger}\right)\right\}\right]$, $\mathbf{R}_{s p}=\mathbf{G}_{s p} \mathbf{x}_{s}\left(\mathbf{G}_{s p} \mathbf{x}_{s}\right)^{\dagger}+\left(\sigma_{n}+\sigma_{e}\right) \mathbf{I}_{N_{p}}$, with $\sigma_{n}^{2}$ the variance of the AWGN at the Rx, uniform power allocation at the Tx $\left(\boldsymbol{\Phi}_{p}=\frac{1}{M_{p}} \mathbf{I}_{M_{p}}\right)$ and single user antenna $(N=1)$.

Fig 6 illustrates the DL channel capacity with beamformers. The SU precoder $\mathbf{P}_{s}$ compensates the $\mathrm{SU}$ interferences, whereas $\mathbf{P}_{p}$ improves the PU transmission with CSIT in low SNR region (see $C_{p}$ : Int, No-Prec; $C_{p}$ : Prec+Int). In the high SNR region, the interferences from PU to SU are not well compensated $\left(M_{p}=10\right)$, and decrease the SU capacity (see $C_{s}$ : $C R$ Prectint), also the contribution of PU precoder is affected by the channel estimation error $\sigma_{e}^{2}=10^{-3}$ (see $C_{p}$ : Prec + int). But as expected, the precoding scheme enhances the PU/SU capacity when the number of antennas grows (see Fig 8). However, as mentioned in [3] and illustrated in Fig. 7 where we vary the SU Tx-power, when the crosslink Tx power increases the performances collapse (see $C_{p}: M M$ Prec), but the SU beamformer solves this problem and keeps compensating SU interferences (see $C_{p}$ : Int CR-prec) even when $\Phi_{s}$ increases. Nonetheless, these performances depend on the crosslink channel estimation accuracy. Fig.7 shows that we can increase the Tx-power (so the capacity) at the $S_{B S}$, with 


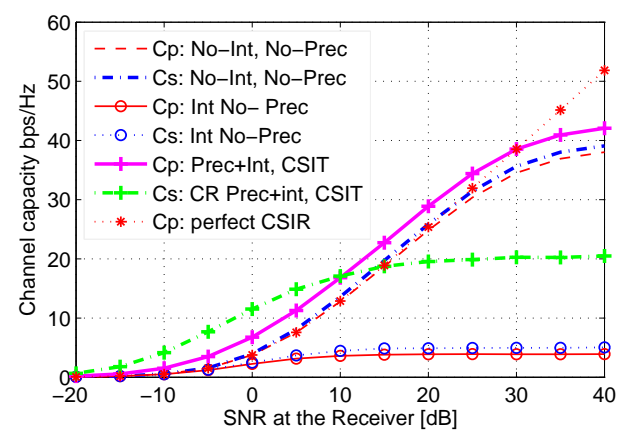

Fig. 6. PU/SU DL channel capacity according to the SNR, $K=L=4$ users, the Tx power $\Phi_{p}=\Phi_{s}=1, M_{p}=10, M_{s}=50, \sigma_{e}^{2}=10^{-3}$.

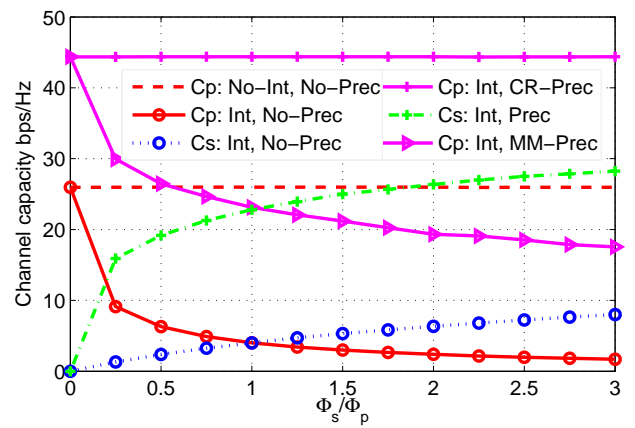

Fig. 7. DL channel capacity versus the SU over PU Tx power $L=K=4$, $M_{p}=M_{s}=100$, with SNR $=20 d B, \sigma_{e}^{2}=10^{-3}$.

minimal disturbance on PU (underlay CR approach). In Fig. 8, we assume all the calibration matrices estimated with an error $\mathbf{n}_{c} \sim \mathcal{C N}\left\{\mathbf{0}, \sigma_{c}^{2} \mathbf{I}\right\}$, then the crosslink channel is found using equations (6) (12). We observe that the DL estimation error grows according to the calibration error and when $\mathbf{n}_{c}$ is high $\left(\Phi / \sigma_{c}^{2} \leq 0 d B, \Phi_{p}=\Phi_{s}=\Phi\right)$, the precoders follow a random beamforming pattern. If $0 d B<\Phi / \sigma_{c}^{2}<10 d B, \mathbf{P}_{s}$ generates additional interferences on PU, though this perturbation is not observed when PU/SU both use a conventional massive MIMO precoder [5], however our precoders improve the PU capacity without increasing the number of antennas. Furthermore Fig. 8 illustrates the benefit provided by SU precoder when $\sigma_{c}^{2} \leq 10^{-1.5}$ for $M=100$ and $\sigma_{c}^{2} \leq 10^{-2}$ for $M=200$. Consequently, a trade-off between the number of antennas, the performances of calibration algorithms and the Tx power are under investigation. It will be discussed in an upcoming study with several PU/SU pairs and different channel models.

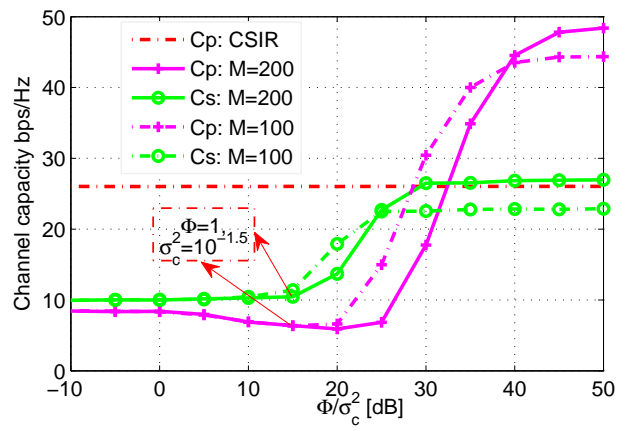

Fig. 8. PU/SU DL capacity according to $\Phi_{p} / \sigma_{c}^{2}$ (the calibration error), with $L=K=4$ and SNR $=20 d B, \sigma_{e}^{2}=10^{-3}, \Phi_{p}=\Phi_{s}=1$.

\section{CONCLUSions}

In this paper, we propose a reciprocity-based CR beamforming exploiting the crosslink channel estimation and the DL channel at the Tx, both determined through a per-user reciprocity-calibration. Simulation results show that the CR precoding scheme reduces considerably the interference from $\mathrm{SU}$ to PU, and large antenna array reduce the uncorrelated interference generated by PU on SU link. However, these improvements depend on the reciprocity calibration efficiency and the CSI accuracy. Finally, this work addresses a practical issue of massive MIMO, namely the reciprocity assumption, and the per-user approach provides as well the basis for further investigations on calibration in MU massive MIMO with mutual coupling at the two ends of the link.

\section{REFERENCES}

[1] A. Goldsmith, S.A. Jafar, I. Maric, and S. Srinivasa, "Breaking spectrum gridlock with cognitive radios: An information theoretic perspective," Proceedings of the IEEE, vol. 97, no. 5, pp. 894-914, 2009.

[2] T.L. Marzetta, "How much training is required for multiuser mimo?," in ACSSC. Asilomar Conf. on, 2006, pp. 359-363.

[3] H.Q. Ngo, T.Q. Duong, and E.G. Larsson, "Uplink performance analysis of multicell mu-mimo with zero-forcing receivers and perfect csi," in IEEE Swe-CTW, 2011, pp. 40-45.

[4] J. Hoydis, S. Ten Brink, and M. Debbah, "Massive mimo: How many antennas do we need?," in 49th Annual Conf. on CCC Allerton, 2011.

[5] T.L. Marzetta, "Noncooperative cellular wireless with unlimited numbers of base station antennas," Wireless Communications, IEEE Trans. on, vol. 9, no. 11, pp. 3590-3600, 2010.

[6] H. Huh, G. Caire, H.C. Papadopoulos, and S.A. Ramprashad, "Achieving large spectral efficiency with tdd and not-so-many base-station antennas," in APWC, IEEE-APS Topical Conf. on, 2011, pp. 1346-1349.

[7] H. Krim and M. Viberg, "Two decades of array signal processing research: the parametric approach," IEEE Signal Processing Magazine, vol. 13, no. 4, pp. 67-94, 1996.

[8] F. Negro, I. Ghauri, and D.T.M. Slock, "Transmission techniques and channel estimation for Spatial Interweave TDD Cognitive Radio systems," in 43rd ACSSC. Asilomar Conf. on, 2009, pp. 523-527.

[9] M. Guillaud, D.T.M. Slock, and R. Knopp, "A practical method for wireless channel reciprocity exploitation through relative calibration," 8th ISSPA, Australia, pp. 403-406, 2005.

[10] B. Kouassi, I. Ghauri, B. Zayen, and L. Deneire, "On the performance of calibration techniques for cognitive radio systems," in The 14th International Symp. WPMC, France, 2011.

[11] B. Kouassi, I. Ghauri, and L. Deneire, "Estimation of Time-Domain Calibration Parameters to Restore MIMO-TDD Channel Reciprocity," in 7th ICST International Conf. CROWNCOM, Sweden, 2012.

[12] B. Kouassi, B. Zayen, R. Knopp, F. Kaltenberger, D. Slock, I. Ghauri, F. Negro, and L. Deneire, "Design and implementation of spatial interweave lte-tdd cognitive radio communication on an experimental platform," IEEE Wireless Communications Magazine, 2013.

[13] C. Shepard, H. Yu, N. Anand, E. Li, T. Marzetta, R. Yang, and L. Zhong, "Argos: practical many-antenna base stations," in Proceedings of the 18th ACM International Conf. MOBICOM, Turkey, 2012, pp. 53-64.

[14] L. Zheng and D.N.C. Tse, "Diversity and multiplexing: A fundamental tradeoff in multiple-antenna channels," Inf. Theory, IEEE Trans. on, vol. 49, no. 5, pp. 1073-1096, 2003.

[15] S.A. Jafar and M.J. Fakhereddin, "Degrees of freedom for the mimo interference channel," Inf. Theory, IEEE Trans. on, vol. 53, no. 7, 2007.

[16] S.M. Kay, Fundamentals of Statistical Signal Processing: Estimation Theory, Prentice Hall Signal Processing Series, vol. 1. 1993.

[17] S. Durrani and M.E. Bialkowski, "Effect of mutual coupling on the interference rejection capabilities of linear and circular arrays in cdma systems," Ant. and Prop., IEEE Trans. on, vol. 52, no. 4, 2004.

[18] I. Markovsky and S. Van Huffel, "Overview of total least-squares methods," Signal processing, vol. 87, no. 10, pp. 2283-2302, 2007.

[19] T.L. Marzetta and B.M. Hochwald, "Fast transfer of channel state information in wireless systems," Signal Processing, IEEE Trans. on, vol. 54, no. 4, pp. 1268-1278, 2006. 